\title{
Efficient Median Finding and Its Application to Two-Variable Linear Programming on Mesh-Connected Computers with Multiple Broadcasting*
}

\author{
Yen-Cheng Chen and Wen-Tsuen Chen \\ Department of Computer Science, National Tsing Hua University, Hsinchu, Taiwan, 30043, Republic of China
}

AND

\section{Gen-Huey CheN}

Department of Computer Science and Information Engineering, National Taiwan University, Taipei, Taiwan, Republic of China

This paper considers 2-dimensional mesh-connected computers with multiple broadcasting (2-MCCMBs). A 2-MCCMB is constructed by augmenting a 2-dimensional mesh-connected computer with broadcasting features in each row and each column. One property of the modified mesh is that rectangular 2MCCMBs, instead of square ones, are the best form for several algorithms. For the median problem of $N$ numbers, a previous algorithm with time complexity $O\left(N^{1 / 6}(\log N)^{2 / 3}\right)$ was developed on an $N^{1 / 2} \times N^{1 / 2} 2$-MCCMB. This paper shows that the time complexity can be reduced to $O\left(N^{1 / 8} \log N\right)$ if an $N^{5 / 8} \times N^{3 / 8}$ rectangular 2-MCCMB is used. Using the above result, we further develop algorithms for two-variable linear programming on an $N^{5 / 8} \times N^{3 / 8} 2-\mathrm{MCCMB}$, where $N$ is the number of constraints. In addition, to achieve better performance on the $N^{5 / 8} \times N^{3 / 8} 2$ MCCMB, we further modify a previous prune-and-search strategy for solving the problem and eventually obtain a parallel algorithm with $O\left(N^{1 / 8} \log N\right)$ time on an $N^{5 / 8} \times N^{3 / 8} 2$-MCCMB. 1992 Academic Press, Inc.

\section{INTRODUCTION}

Mesh-connected computers (MCCs) have been widely used because their regular structure and simple interconnection are quite suitable for VLSI implementation. A 2dimensional mesh-connected computer (2-MCC) of size $N$ consists of an $m \times n$ array of processing elements (PEs), where $m \times n=N$. Very often, $m=n=N^{1 / 2}[5$, 12, 16]. Each PE is connected to its four nearest neighbors, provided they exist. As in Nassimi and Sahni [13], it is assumed that a PE may transmit unit data to any of its four nearest neighbors in unit time. Moreover, each PE has some local memory.

The regular pattern of the mesh-connected network makes MCCs suitable for solving many problems from

\footnotetext{
* This work was supported by the National Science Council, Taiwan, Republic of China, under Grant NSC79-0408-E007-18.
}

matrix manipulation and image processing [5, 12]. However, its local connectivity nature will result in a long communication delay when data have to be moved over a long distance. Thus, their large diameter is a main shortcoming of MCCs. To overcome the communication inefficiency, several authors have augmented MCCs with various faster mechanisms $[1,3,4,6,7,10,14,15]$. In some of them, the capability of long distance communications is enhanced through the use of one or more broadcast buses. Several variants of MCCs with broadcasting features were thus proposed $[1,4,10,14,15]$. Indeed, many problems can be solved more efficiently on these modified MCCs. The modified meshes discussed here are 2dimensional mesh-connected computers with multiple broadcasting (2-MCCMBs) [10]. A 2-MCCMB is a 2dimensional mesh-connected computer with a bus for each row and column. That is, the PEs in the same row or column are connected to a bus in addition to the local links, as shown in Fig. 1. Hence, there are two types of data transfers executed by each PE of a 2-MCCMB: routing data into one of its four nearest neighbors via a local link and broadcasting data to the other PEs in the same row (or column) via the bus on this row (or column). As in Kumar and Raghavendra [10] and Stout [15], it is assumed that broadcasting takes constant time and only one PE is permitted to broadcast data on each row bus and each column bus at a time. However, to avoid the overhead caused by conflicts, all algorithms on MCCMBs are required to be conflict-free [10, 15]. That is, parallel algorithms on MCCMBs must be developed so that no two PEs attempt to use the same bus at the same time.

Very often, 2-MCCs are configured as squares in order to easily map applications and data onto them. Square 2MCCs achieve the minimal diameter so that better performance can be obtained. Therefore, it is natural to want to map applications onto square 2-MCCMBs. However, 
because of the augmented broadcasting feature, the diameter consideration is not necessary any more for 2MCCMBs. This makes possible the use of rectangular 2MCCMBs to achieve better performance. By using rectangular 2-MCCMBs, Chen et al. [8] have successfully improved Kumar and Raghavendra's algorithm [10] for semigroup computations of $N$ data items. They showed that $N^{5 / 8} \times N^{3 / 8}$ rectangular 2-MCCMBs are the optimal form for the algorithm and the time complexity can be reduced from $O\left(N^{1 / 6}\right)$ to $O\left(N^{1 / 8}\right)$. Therefore, a feasible way to obtain a more efficient algorithm on 2MCCMBs is by adjusting the dimensions of MCCMBs to match the communication patterns of the algorithm. In this paper, we shall apply this strategy to two algorithms more complicated than the one for semigroup computations. First, in Section 2 it will be seen that finding the median of $N$ values takes $O\left(N^{1 / 8} \log N\right)$ time on an $N^{5 / 8} \times$ $N^{3 / 8}$ rectangular 2-MCCMB. This improves the previously best known result of $O\left(N^{1 / 6}(\log N)^{2 / 3}\right)$ time on an $N^{1 / 2} \times N^{1 / 2} 2$-MCCMB. The second problem discussed is two-variable linear programming. An elegant prune-andsearch strategy for solving the problem has been proposed in [11]. To adopt this strategy on 2-MCCMBs, we find that an efficient constraint pairing operation, i.e., arranging the constraints into pairs, is a prerequisite. It will be seen in Section 3 that this operation can be performed in $O\left(N^{1 / 8}\right)$ time on an $N^{5 / 8} \times N^{3 / 8} 2$-MCCMB, where $N$ is the number of constraints. Applying the above results for constraint pairing and median finding, we develop an $O\left(N^{1 / 8}(\log N)^{2}\right)$ time algorithm for the linear programming problem with $N$ constraints on an $N^{5 / 8} \times N^{3 / 8} 2$-MCCMB. However, we further find that this result is not good enough when applying the pruneand-search strategy directly. In the $O\left(N^{1 / 8}(\log N)^{2}\right)$ time algorithm, the time required in each iteration is dominated by the median finding operation. Section 3 will show that the step performing a median finding operation can be modified so that each iteration takes at most $O\left(N^{1 / 8}\right)$ time. Consequently, an algorithm with time complexity $O\left(N^{1 / 8} \log N\right)$ is obtained by applying the modificd prunc-and-search strategy.

\section{MEDIAN PROBLEM}

Given $N$ elements distributed over a 2-MCCMB of size $N$ with one element per PE, the median problem is to find the median value of the $N$ elements. The strategy we adopt is a prune-and-search one proposed in a linear time median finding algorithm [2]. It consists of $O(\log N)$ iterations, and in each iteration a partition element $m$ is first determined to partition the set of possible candidates, i.e., the elements which are not yet pruned away, into two subsets. Each subset is guaranteed to be a constant fraction of the size of the set of candidates. If the rank of

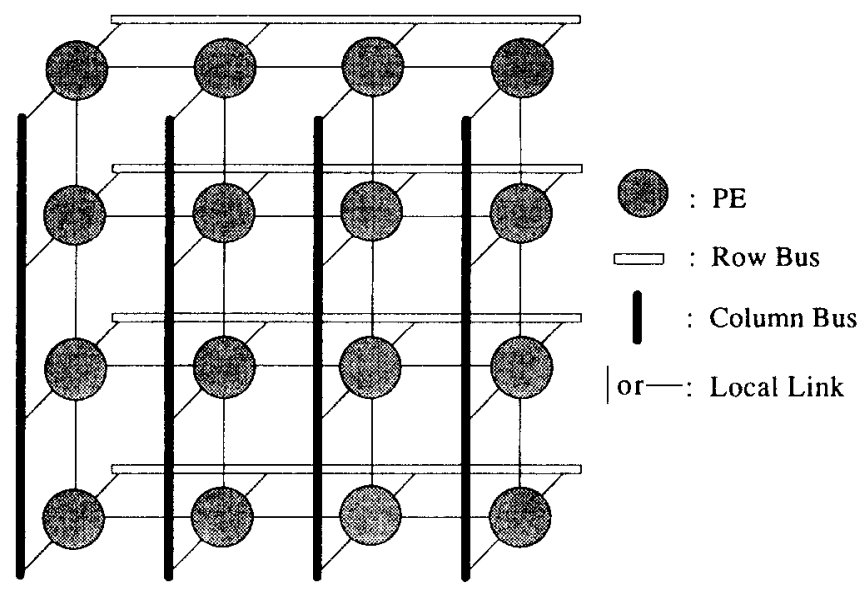

FIG. 1. A $4 \times 4$ 2-dimensional mesh-connected computer with multiple broadcasting.

$m$ is $N / 2$, then $m$ is the median value. Otherwise, one of the subsets, and thus a constant fraction of the set of candidates, can be pruned away. Hence, in $O(\log N)$ iterations, the median value can be found. In Kumar and Raghavendra [10], it has been shown that median finding can be performed in $O\left(N^{1 / 6}(\log N)^{2 / 3}\right)$ time on an $N^{1 / 2} \times$ $N^{1 / 2}$ 2-MCCMB. In the following, we shall develop a median finding algorithm with time complexity $O\left(N^{1 / 8} \log \right.$ $N)$ on the $N^{5 / 8} \times N^{3 / 8}$ rectangular 2-MCCMB.

The $N^{5 / 8} \times N^{3 / 8} 2$-MCCMB is first partitioned into disjoint $N^{1 / 8} \times N^{1 / 8}$ submeshes, called blocks. These blocks form an $N^{1 / 2} \times N^{1 / 4}$ array. Denote block $(i, j)$ as the block at location $(i, j)$ of the array. Furthermore, a row of blocks is called a band. We further partition each band into $N^{1 / 8}$ groups, each containing $N^{1 / 8}$ contiguous blocks. In addition, $N^{1 / 8}$ contiguous bands form a band-group. Refer to Fig. 2. Under this partitioning, each band contains $N^{1 / 8}$ groups and $N^{1 / 8}$ row buses. Therefore, each group can be assigned a dedicated row bus. Moreover, it is also known that there are $N^{3 / 8}$ band-groups and $N^{3 / 8}$ column buses. Similarly, each band-group can be assigned a dedicated column bus. In our algorithm, data communication within a group (band-group) will be performed via a row (column) bus. The upper left PE of cach block (band) is said to be the block (band) leader. A PE is said to be active if it contains a candidate for the median value, and the candidate is called an active element. That is, the active elements are those which are not yet pruned away. For easy description, the following notations are defined.

$\operatorname{act}(i, j)=$ number of active elements in block $(i, j)$. $m(i, j)=$ the median of the active elements in block $(i, j)$.

act $(i)=$ number of active elements in band $i$. 
Block : $\mathrm{N}^{1 / 8} \times \mathrm{N}^{1 / 8}$ PEs; $\quad \mathrm{N}^{1 / 2} \times \mathrm{N}^{1 / 4}$ blocks

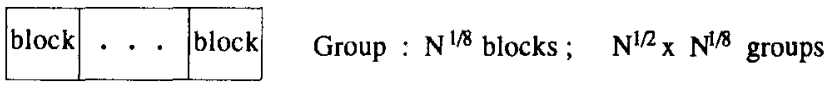

\begin{tabular}{|c|c|c|}
\hline group & $\cdot \cdot \cdot$ & group \\
\hline \multicolumn{2}{|c|}{ Band : $\mathrm{N}^{1 / 8}$ groups ; $\mathrm{N}^{1 / 2}$ bands }
\end{tabular}

\begin{tabular}{|c|}
\hline band \\
\hline$\dot{\vdots}$ \\
\hline band \\
\hline
\end{tabular}

Band-group : $\mathrm{N}^{1 / 8}$ bands ; $\quad \mathrm{N}^{3 / 8}$ band-groups

FIG. 2. Blocks, groups, bands, and band-groups of an $N^{5 / 8} \times N^{3 / 8}$ 2-MCCMB.

$m \omega=$ the weighted median $m(i, s)$, for some $s$, in band $i$ with

$\left.\sum_{j} \operatorname{act}(i, j)\right|_{m(i, j)<m(i, s)}<\operatorname{act}(i) / 2$

and $\left.\sum_{j} \operatorname{act}(i, j)\right|_{m(i, j) \leq m(i, s)} \geq \operatorname{act}(i) / 2$,

where $\left.\operatorname{act}(i, j)\right|_{m(i, j)<(\leq) m(i, s)}$ means the $\operatorname{act}(i, j)$ with $m(i, j)<(\leq) m(i, s)$.

$a c t=$ number of active elements.

$m=$ the weighted median $m(t)$, for some $t$, with $\Sigma_{i}$ $\left.\operatorname{act}(i)\right|_{m(i)<m(t)}<\operatorname{act} / 2$ and

$$
\left.\sum_{i} a c t(i)\right|_{m(i) \leq m(t)} \geq a c t / 2 .
$$

$\operatorname{rank}(m)=$ rank of $m$ among all elements.

Furthermore, let $S_{1}\left(S_{2}\right)$ be the number of active elements $<(>) m$. Initially let all PEs be active. A median finding algorithm on an $N^{5 / 8} \times N^{3 / 8} 2$-MCCMB is as follows.

Step 1. Sort the data in each block via local links. This step takes $O\left(N^{1 / 8}\right)$ time [16].

Step 2. Compute $\operatorname{act}(i, j)$ and $m(i, j)$ for each block $(i, j)$.

In each block $(i, j)$, there is a least-numbered $\mathrm{PE}$ and a greatest-numbered $\mathrm{PE}$ which are active during an itera- tion. Since the elements in each block have been sorted, we can easily determine $a c t(i, j)$ according to the indices of these two PEs in block $(i, j)$. Moreover, we can also determine which active PE contains $m(i, j)$. The median $m(i, j)$ is sent to the block leader of block $(i, j)$. The time requirement of Step 2 is $O\left(N^{1 / 8}\right)$.

Step 3. Compute act $(i)$ and $m(i)$ for each band $i$.

Since $\operatorname{act}(i)=\sum_{j} \operatorname{act}(i, j)$, it is required for each band to sum $N^{2 / 8}$ values each stored in a block leader. This can be performed by first summing $N^{1 / 8}$ act $(i, j)$ 's within each group via row broadcasting. Then, the summation of the $N^{1 / 8}$ computed sums, which are stored in the left-most column of the band, is performed via local links. Therefore, act $(i)$ can be determined in $O\left(N^{1 / 8}\right)$ time. Also, during the computation of act $(i)$, the block leader of block $(i, j)$ can compute $\left.\Sigma_{k} \operatorname{act}(i, k)\right|_{m(i, k)<m(i, j)}$ and $\Sigma_{k}$ act $\left.(i, k)\right|_{m(i, k) \leq m(i, j)}$. Hence, $m(i)$ can be also determined in $O\left(N^{1 / 8}\right)$ time. The weighted median $m(i)$ is stored in the band leader of band $i$.

Step 4. Compute act and $m$.

The computation of act and $m$ is similar to Step 3. The summation of $N^{1 / 2}$ act $(i)$ 's can be performed first via column buses (for summing $N^{1 / 8}$ act $(i$ )'s within each band-group) and then via the row buses and local links of the first band (for summing the $M^{3 / 8}$ computed sums). Hence, act can be determined in $O\left(N^{1 / 8}\right)$ time. Also, during the computation of $a c t$, the band leader of band $i$ can compute $\left.\sum_{k} \operatorname{act}(k)\right|_{m(k)<m(i)}$ and $\left.\sum_{k} \operatorname{act}(k)\right|_{m(k) \leqslant m(i)}$. Hence, in $O\left(N^{1 / 8}\right)$ time, $m$ can be also determined.

Step 5. Compute $\operatorname{rank}(m)$. If $\operatorname{rank}(m)=N / 2$ then $m$ is the median value. Otherwise, if $\operatorname{rank}(m)<(>) N / 2$, let the elements of $S_{1}\left(S_{2}\right)$ become passive and go to Step 2.

The computation of $\operatorname{rank}(m)$ is similar to a semigroup computation. At first, $m$ is broadcast to each PE. Each PE then compares $m$ with its own element. If $m$ is greater, set $r=1$; otherwise, set $r=0$. Finally, compute the sum of the $r$ 's. The sum is equal to $\operatorname{rank}(m)$. Therefore, this step takes $O\left(N^{1 / 8}\right)$ time.

Since in each block $(i, j)$, half of its active elements are $\leq(\geq) m(i, j)$, at least a quarter of the active elements in band $i$ are $\leq(\geq) m(i)$. This further implies that at least $1 / 8$ of all active elements are $\leq(\geq) m$; i.e., $\left|S_{1}\right|,\left|S_{2}\right| \geq$ act/8. Therefore, the median value can be found in $O(\log N)$ iterations. Since each iteration takes $O\left(N^{1 / 8}\right)$ time, the total time complexity is $O\left(N^{1 / 8} \log N\right)$. Hence, we have the following theorem.

THEOREM 1. The median of $N$ values can be determined in $O\left(N^{1 / 8} \log N\right)$ time on an $N^{5 / 8} \times N^{3 / 8}$ rectangular 2-MCCMB.

The above algorithm improves the previously best known result on square 2-MCCMBs [10]. In Kumar and 
Raghavendra's algorithm [10], the square $N^{1 / 2} \times N^{1 / 2} 2$ MCCMB is first partitioned into disjoint blocks with size $N^{1 / 6}(\log N)^{2 / 3} \times N^{1 / 6}(\log N)^{2 / 3}$. Under this partitioning, to move data within each such block to the block leader in $O\left(N^{1 / 6} /(\log N)^{1 / 3}\right)$ time, an additional algorithm, called Roundup, was developed. This is not required for our algorithm. Besides, in our algorithm, using rectangular 2MCCMBs leads to more available row buses. Since there is more communication capability in broadcasting, the amount of communication relying on local links can be decreased. Further, when the local and broadcast communications are balanced, the best performance can be achieved. This is the reason why the block size is $N^{1 / 8} \times$ $N^{1 / 8}$.

\section{TWO-VARIABLE LINEAR PROGRAMMING}

A linear programming problem with two variables can be defined as

Minimize $c_{1} x_{1}+c_{2} x_{2}$

Subject to $a_{i 1} x_{1}+a_{i 2} x_{2} \geq \beta_{i} \quad(i=1,2, \ldots, N)$.

We adopt the prune-and-search strategy used in Megiddo's linear time sequential algorithm for the problem [11]. The basic idea of the prune-and-search strategy is that there are always some constraints which do not affect the solution and therefore can be pruned away. Hence, if a constant fraction of constraints is pruned away after each iteration, the linear programming problem can be solved in $O(\log N)$ iterations.

For convenience, (1) is first transformed into the form

$$
\begin{array}{ll}
\text { Minimize } & y \\
\text { subject to } & y \geq a_{i} x+b_{i} \quad\left(i \in I_{1}\right) \\
& y \leq a_{i} x+b_{i} \quad\left(i \in I_{2}\right) \\
& a \leq x \leq b,
\end{array}
$$

where $\left|I_{1}\right|+\left|I_{2}\right| \leq N$ and $-\infty \leq a, b \leq \infty$. Furthermore, define $g(x)=\max \left\{a_{i} x+b_{i}: i \in I_{1}\right\}$ and $h(x)=\min \left\{a_{i} x+\right.$ $\left.b_{i}: i \in I_{2}\right\}$. In Megiddo's algorithm, a point $x, a \leq x \leq b$, is first carefully selected and the following values are then computed.

$$
\begin{aligned}
& s_{g}(x)=\min \left\{a_{i}: i \in I_{1}, a_{i} x+b_{i}=g(x)\right\} \\
& S_{g}(x)=\max \left\{a_{i}: i \in I_{1}, a_{i} x+b_{i}=g(x)\right\} \\
& s_{h}(x)=\min \left\{a_{i}: i \in I_{2}, a_{i} x+b_{i}=h(x)\right\} \\
& S_{h}(x)=\max \left\{a_{i}: i \in I_{2}, a_{i} x+b_{i}=h(x)\right\} .
\end{aligned}
$$

Let $x^{*}$ be the optimum solution. According to the above values, we can test the following:
Case 1. $g(x) \leq h(x)$ (feasible case)

(a) If $s_{g}(x)>0$ and $S_{g}(x)>0$, then $x^{*}<x$.

(b) If $s_{g}(x)<0$ and $S_{g}(x)<0$, then $x^{*}>x$.

(c) If $s_{g}(x)<0$ and $S_{g}(x)>0$, then $x$ is the optimum solution.

Case 2. $g(x)>h(x)$ (infeasible case)

(a) If $s_{g}(x)>S_{h}(x)$, then $x^{*}<x$.

(b) If $S_{g}(x)<s_{h}(x)$, then $x^{*}>x$.

(c) If $s_{g}(x) \leq S_{h}(x)$ and $S_{g}(x) \geq s_{h}(x)$, then no feasible solution exists.

Therefore, we can either find that the problem is infeasible, recognize that $x$ itself is the optimum solution, or decide that the range in which $x^{*}$ may exist is confined to one of the subintervals $[a, x],[x, b]$. Following the above test is the pruning of redundant constraints. Given a pair of constraints, the subinterval containing $x^{*}$ is used to determine which constraint of the pair is redundant. For the overall detailed description, the interested reader may consult Megiddo [11]. To guarantee that a constant fraction of constraints are pruned away during each iteration, a median finding operation is included in Megiddo's algorithm for the previous selection of $x$. The algorithm can be summarized as follows.

Step 1. Arrange the constraints in $I_{1}$ and $I_{2}$ into arbitrary disjoint pairs. For each pair $i, j$, if $a_{i}=a_{j}$ (i.e., parallel), drop one redundant constraint. Otherwise, find the intersection $\left(x_{i j}, y_{i j}\right)$ of $y=a_{i} x+b_{i}$ and $y=a_{j} x+b_{j}$.

Step 2. Find the median $x_{m}$ of the $x_{i j}$ 's.

Step 3. Compute $g\left(x_{m}\right), h\left(x_{m}\right), s_{g}\left(x_{m}\right), S_{g}\left(x_{m}\right), s_{h}\left(x_{m}\right)$, and $S_{h}\left(x_{m}\right)$.

(a) If $x_{m}$ is optimal, report this and exit.

(b) If no feasible solution exists, report this and exit.

(c) Otherwise, determine whether $x^{*}$ lies to the left, or right, of $x_{m}$.

Step 4. Discard at least $1 / 4$ of the constraints. Go to Step 1.

Now we discuss how the above operations are performed on an $N^{5 / 8} \times N^{3 / 8}$ 2-MCCMB. As shown in Fig. 2, the 2-MCCMB is conceptually partitioned into blocks, groups, bands, and band-groups. Initially each PE holds one constraint. Before Step 1, a form transformation (from (1) to (2)) is first performed to the constraint in each PE. This takes $O\left(N^{1 / 8}\right)$ time [9]. In Step 1, constraint pairing is performed on $I_{1}$ and $I_{2}$ independently. The constraint pairing resembles a semigroup computation. At first, pairing is performed within each block. Since the number of constraints in each block is either even or odd, there will be at most one constraint that remains unpaired 
in each block. Then, pair unpaired constraints in each group. The subsequent pairing operations are then performed in each band and band-group. Finally, the overall constraint pairing can be completed by one additional pairing among all band-groups. For more details, consult Chen et al. [9]. The hierarchical pairing thus takes $O\left(N^{1 / 8}\right)$ time. Step 2 is a median finding operation, which takes $O\left(N^{1 / 8} \log N\right)$ time (Theorem 1). Step 3 is a series of semigroup computations. This can be done in $O\left(N^{1 / 8}\right)$ time [9]. Therefore, each iteration is of order $O\left(N^{1 / 8} \log \right.$ $N$ ), which is dominated by the median finding operation. Thus the linear programming problem can be solved in $O\left(N^{1 / 8}(\log N)^{2}\right)$ time on an $N^{5 / 8} \times N^{3 / 8} 2$-MCCMB. However, this time complexity can be further reduced by modifying the above prune-and-search strategy.

THEOREM 2. Two-variable linear programming with $N$ constraints can be solved in $O\left(N^{1 / 8} \log N\right)$ time on an $N^{5 / 8} \times N^{3 / 8} 2-M C C M B$ with each PE holding one constraint initially.

Proof. In the above algorithm, finding the median value of intersections guarantees that at least one-quarter of constraints can be pruned away in each iteration. In fact, it is not necessary to find the precise median value. We find that the weighted median $m$ computed in the first iteration of the median finding algorithm is adequate for the purpose of pruning. By our modification, i.e., replacing Step 2 by performing only one iteration of the median finding algorithm, at least $1 / 16$ of constaints can be dropped in each itcration. It is still guaranteed that the linear programming problem can be solved in $O(\log N)$ iterations. However, each step of the modified algorithm takes at most $O\left(N^{1 / 8}\right)$ time. Therefore, a new algorithm with time complexity $O\left(N^{1 / 8} \log N\right)$ is attained. Q.E.D.

\section{DISCUSSION}

In Section 2 and 3, efficient parallel algorithms have been successfully derived by adopting an $N^{5 / 8} \times N^{3 / 8}$ rectangular 2-MCCMB. In general, the $N^{5 / 8} \times N^{3 / 8}$ rectangular 2-MCCMB is a good candidate for designing parallel algorithms that are based on tree-like or prefix computations. Theoretically, we may carefully choose the best form of the adopted 2-MCCMB to achieve better performance for a parallel algorithm. In practice, to get integral values, the size of $N$ is restricted. For example, $N$ must be equal to $256,6561,64 \mathrm{~K}, \ldots$ (i.c., of the form $a^{8}$, where $a=2,3, \ldots)$ for the above algorithms. This limits the scope of our results. However, our approach can be generalized to arbitrary $X \times Y 2$-MCCMBs with $X Y=N$, so that given any $2-\mathrm{MCCMB}$ of size $N$, we can derive efficient algorithms for it. Without loss of generality, let $X \geq Y$. Assume $p=\left[\max \left\{Y^{1 / 3}, N^{1 / 2} / Y\right\}\right]$. Let block size be $p \times p$, group size be $p \times Y / p$, band size be $p \times Y$, and band-group size be $X / Y \times Y$. Under the partitioning, it can be verified, without much effort, that the median finding takes $O(p \log N)$ time. Further, two-variable linear programming can be performed in $O(p \log N)$ time on an $X \times Y 2$-MCCMB. Therefore, we propose the generalized approach to achicving better performance for a given arbitrary 2-MCCMB of size $N$. Note that for the median problem and two-variable linear programming the $N^{5 / 8} \times N^{3 / 8}$ rectangular $2-\mathrm{MCCMB}$ is the one with the best performance among the 2-MCCMBs of size $N$.

\section{CONCLUDING REMARKS}

Adopting rectangular 2-MCCMBs results in more communication capability in broadcasting. Further, when the local and broadcast communication is balanced, the best performance can be achieved. Thus, this paper shows that square 2-MCCMBs are not favorable for the median problem. The previous algorithm for median finding on square 2-MCCMBs was improved by using an $N^{5 / 8} \times N^{3 / 8}$ rectangular 2-MCCMB. The time complexity was reduced to $O\left(N^{1 / 8} \log N\right)$, vs $O\left(N^{1 / 6}(\log N)^{2 / 3}\right)$ on an $N^{1 / 2} \times$ $N^{1 / 2}$ 2-MCCMB. We also discussed two-variable linear programming on an $N^{5 / 8} \times N^{3 / 8} 2$-MCCMB. This problem can be regarded as a more complicated application on 2MCCMBs. To obtain a more efficient algorithm, we modified Megiddo's prune-and-search strategy and successfully reduced the time required in each iteration from $O\left(N^{1 / 8} \log N\right)$ to $O\left(N^{1 / 8}\right)$. Therefore, a parallel algorithm with time complexity $O\left(N^{1 / 8} \log N\right)$ was obtained.

\section{REFERENCES}

1. Aggarwal, A. Optimal bounds for finding maximum on array of processors with $k$ Global buses. IEEE Trans. Comput. C 35, 1 (Jan. 1986), 62-64.

2. Aho, A. V., Hopcroft, J. E., and Ullman, J. D. The Design and Analysis of Computer Algorithms. Addison-Wesley, Reading, MA, 1974.

3. Bokhari, S. H. MAX: An algorithm for finding maximum in an array processor with a Global Bus. Proc. 1981 International Conference on Parallel Processing, 1981, pp. 302-303.

4. Bokhari, S. H. Finding maximum on an array processor with a global bus. IEEE Trans. Comput. C 33, 2 (Feb. 1984), 133-139.

5. Cappello, P. R. A mesh automation for solving dense linear systems. Proc. 1985 International Conference on Parallel Processing, Aug. 1985, pp. 418-425.

6. Carlson, D. A. Performing tree and prefix computations on modified mesh-connected parallel computers. Proc. 1985 International Conference on Parallel Processing, Aug. 1985, pp. 715-718.

7. Carlson, D. A. Solving linear recurrence systems on meshconnected computers with multiple global buses. J. Parallel Distrib. Comput. 8, 1990, 89-95.

8. Chen, Y. C., Chen, W. T., Chen, G. H., and Sheu, J. P. Designing efficient parallel algorithms on mesh-connected computers with multiple broadcasting. IEEE Trans. Parallel Distrib. Systems 1, 2 (Apr. 1990), 241-246. 
9. Chen, Y. C., Chen, W. T., and Chen, G. H. Parallel algorithms for two-variable linear programming on mesh-connected computers with multiple broadcasting. Tech. Rep, Department of Computer Science, National Tsing Hua University, Hsinchu, Taiwan, Republic of China, 1990.

10. Prasanna Kumar, V. K., and Raghavendra, C. S. Array processor with multiple broadcasting. J. Parallel Distrib. Comput. 4 (1987), 173-190.

11. Megiddo, N. Linear-time algorithms for linear programming in $R^{3}$ and related problems. SIAM J. Comput. 12, 4 (Nov. 1983).

12. Miller, R., and Stout, Q. F. Geometric algorithms for digitized pictures on a mesh-connected computer. IEEE Trans. Pattern Anal. Mach. Intell. PAMI-7, 2 (Mar. 1985).

13. Nassimi, D., and Sahni, S. Data broadcasting in SIMD computers. IEEE Trans. Comput. C 30, 2 (Feb. 1981), 101-107.

14. Stout, Q. F., Mesh-connected computers with broadcasting. IEEE Trans. Comput. C 32, 9 (Sept. 1983), 826-830.

15. Stout, Q. F. Meshes with multiple buses. Proc. 27th IEEE Symposium on Foundations of Computer Science, 1986, pp. $264 \ldots 273$.

16. Thompson, C. D., and Kung, H. T. Sorting on a mesh-connected computer. Comm. Assoc. Comput. Mach. 20, 4 (Apr. 1977), 263271.

YEN-CHENG CHEN was born in Taiwan, Republic of China, on December 13, 1963. He received the B.S. degree in electrical engineering from National Cheng Kung University, Taiwan, in 1986 and the M.S. degree in information engineering from Tatung Institute of Technology. Taiwan, in 1988. Since September 1988, he has been working

Received August 14, 1990; revised May 1, 1991; accepted August 23, 1991 toward the Ph.D. degree in the Institute of Computer Science of the National Tsing Hua University, Taiwan, Republic of China. His current research interests include parallel algorithms, parallel processing, and multiprocessor systems.

WEN-TSUEN CHEN was born in Taiwan, Republic of China, on May 27, 1948. He received the B.S. degree in nuclear engineering from National Tsing Hua University, Taiwan, and the M.S. and Ph.D. degrees from the University of California, Berkeley, in 1970, 1973, and 1976, respectively. He joined the faculty of the Institute of Computer Science, National Tsing Hua University, in March 1976 as an associate professor. Since 1979, he has been a professor and from 1983 to 1988 he served as the director of the Institute. In 1980, he was a visiting professor in the EECS Department of the University of California, Berkeley. From 1984 to 1985, he held an elected post of IEEE Distinguished Visitor in region 10. Since 1988, he has been a member of the Science and Technology Advisory Board of the Ministry of Education, Taiwan, Republic of China. His current research interests include computer networks, broadband ISDN, multiprocessor systems, and parallel algorithms.

GEN-HUEY CHEN was born in Taiwan, Republic of China, on October 10, 1959. He received the B.S. degree in computer science from National Taiwan University, Taiwan, in June 1981 and the M.S. and Ph.D. degrees in computer science from National Tsing Hua University in June 1983 and January 1987, respectively. In February 1987, he joined the faculty of National Taiwan University and he now is an associate professor of the Department of Computer Science and Information Engineering. His current research interests include design and analysis of algorithms, distributed algorithms, parallel computation, and parallel computer architectures. 